\title{
Monitoring and Controlling Phone Usage to Raise Awareness and Combat Digital Addiction
}

\author{
Klara Potapova \\ Department of Computing \&Informatics \\ Bournemouth University \\ Poole, United Kingdom \\ s4913979@bournemouth.ac.uk
}

\author{
Deniz Cetinkaya \\ Department of Computing\&Informatics \\ Bournemouth University \\ Poole, United Kingdom \\ dcetinkaya@bournemouth.ac.uk
}

\author{
Gernot Liebchen \\ Department of Computing \& Informatics \\ Bournemouth University \\ Poole, United Kingdom \\ gliebchen@bournemouth.ac.uk
}

\begin{abstract}
One of the defining factors in human progress is the fact how humans have adopted technology into their everyday lives. One of these technologies that has seen a tremendous increase in usage is the mobile phone. The potential overuse of a smartphone device is very easily done, with many possible bad psychological side effects. Digital addiction is a form of addiction that has become more prevalent with people due to the ever-growing technological advances that our devices have achieved. This work focuses on what could be done to assist people via a software application who either have the addiction or help prevent people from becoming addicted. This paper presents design and implementation of a mobile application to monitor and control the phone usage so that it can help combat digital addiction. The prototype implementation lets user see how much time they use on their phone as well as set some preferences. The study has been evaluated by user testing and having user feedback.
\end{abstract}

Keywords-digital addiction, mobile applications, phone usage monitoring, social computing

\section{INTRODUCTION}

One of the defining factors in human progress is the fact how humans have adopted technology into their everyday lives, especially those in more advanced economies [1]. One of these technologies that has seen a tremendous increase in usage is the smartphone. Nowadays, phones let a user do almost anything they need without the use of a computer, as well as reel people in with many mobile applications. Digital addiction is a form of addiction that has become more prevalent with people due to the ever-growing technological advances that our devices have achieved [2]. Digital addiction is something that can happen with any user who owns a digital device, such as a smartphone. It is considered someone may be addicted when a user is using their device in an obsessive and compulsive manner [2].

The potential overuse of a smartphone device is very easily done, with many possible bad psychological side effects [3]. Currently, digital addiction is not well known by many people, it can be inferred that not many people are aware that this is something that can affect them. We would like to note that the term digital addiction is still under discussion. It is not a medical term, but it describes a possibly problematic behavioural pattern that may need awareness. Existing tools that assist with controlling phone usage do not necessarily aim to raise any awareness on this topic. Additionally, a lot of these tools require some form of payment in order to use the full features of the tool.

This work focuses on what could be done to assist people via a software application who either have the addiction or help prevent people from becoming addicted. This paper presents design and implementation of a mobile application to monitor and control the phone usage so that it can help combat digital addiction. The prototype implementation lets user see how much time they use on their phone as well as set some preferences. This in turn should promote a healthier and more self-controlled lifestyle for the user by making informed decisions about how much time they spend on their phone. The study has been evaluated by user testing and having user feedback from 13 participants.

The remaining of the paper is structured as follows: Next section provides background information and related work. Section 3 discusses the methods. Section 4 and 5 presents the requirements analysis and design of the proposed approach respectively. Section 6 explains the development details and Section 7 presents the outcome of the evaluation. Finally, last section concludes the paper and discusses the future work.

\section{BACKGROUND}

The impact of someone having digital addiction can be quite severe, on not only the person who is addicted, but their loved ones too. Montag and Walla [4] argue that some people simply stop living life to the fullest when they only live through a screen. This means that a person simply does not enjoy life and in turn becomes obsessed with what is on their screen rather than what is going on in the outside world. They can forget about spending time with others and even ignore people if they are concentrating on their screen too much, even if it is unintentional. This sort of compulsive behaviour results in a myriad of negative effects on a person. It can cause someone to be antisocial; have problems with work performance; become stressed; experience familial disruptions and have possible financial and personal harm [5,6]. This resulting behaviour can often disrupt someone's everyday life, as well as their loved ones who may not experience the same addiction as them. People may lose their jobs due to the lack of productivity or may lose control of their life entirely $[7,8]$. With now over $95 \%$ of all the people in the UK owning a smartphone, it is more likely that a smartphone is one of the devices that has a high risk of being the most common device that people can get addicted to [9].

There are currently a few coping strategies that exist to help alleviate the use of smartphone addiction, however not everyone can do it on their own. Whilst a user may be asked to just set small goals like putting their phone away for an hour or just turn the phone off, this may prove to be very difficult for some if their addiction is severe and they simply cannot put the phone down [2]. Lochtefeld et al. [10] have stated that there is no medication for this addiction yet, so it might be difficult for someone to battle with this. One way in which some help could be provided for the person who is addicted could be from within the phone itself, providing a platform of help from the very same device that they are addicted to. 
There have been a few applications to this day that help a user monitor and control their phone usage to help lessen the time spent on a phone per day. At this current moment, some of the applications that are seen from GooglePlay Store require some form of payment to use most of the useful features [11]. After conducting some research on some current applications that exist for phones such as ActionDash for Android, Digital Wellbeing for Google and Moment for iOS, we analysed the user reviews of each application listed. Based on the analysis of the applications and the reviews, a lot of the existing applications are limited to some form of paywall to access the full features of an application, such as setting a timer for an application or accessing parental settings. It was evident across the different applications that were reviewed that some users were not happy when it came to purchasing premiums or subscriptions for an application of this nature.

Applying monetary requirements from users can deter a user from using an application [11]. This will be counterproductive in the long run, as users might not wish to pay for a feature for the simple reason of wanting to save money. Thus, the user might not be able to monitor and control their phone usage properly or effectively if some features are unavailable due to the user not buying them. This is not ideal for someone who may experience obsessive phone usage behaviours and or have troubles with controlling their excessive phone usage [2]. When we analysed the relevant Android applications and the number of downloads, we have seen that the free version is more favourable by users. Because of this fact, it is important that the solution is free and does not have any paid features that may reduce the potential user base.

For the Digital Wellbeing application from Google, one of the most upvoted reviews was from a user who was unhappy with the fact that the application cannot be uninstalled. This brings up the point of consent and the fact that it is vitally important that a user is given a choice. Cate and MayerSchonberger [12] mention how consent works nowadays in the world of data, and that it "is either non-existent or likely to be so vague as to be meaningless". With such ambiguities, it might cause people to be worried about who can see their personal data. Because of this, it is important that when making an application, the option of letting a user to opt out from having this application on their phone is included. This is especially important when that application will need to look at your phone usage data, which users might be sensitive to.

Ali et al. [13] have distinguished that over $80 \%$ of participants from their study agreed that they would want to be helped with the regulation of their phone usage. This shows intent from users that they wish to see or change their behaviour on their phone regarding usage. The more people making beneficial decisions of using their phones in a noncompulsive manner, the more people there are who have a lower risk of developing digital addiction. Thus, the need for solutions of this kind to assist them with that is evident.

\section{METHODS}

Both quantitative and qualitative research methods were used in this study. Regarding conducting literature and primary research, a deductive approach was taken as it helped review how someone may feel with digital addiction and what sorts of consequences can arise from having this addiction before creating a survey and creating the requirements for the application. Based on the data collected before proper review of literature and primary research, this data can assist with accuracy towards understanding the addiction, as well as eliminate any potential bias that may be included within the solution. Such biases could be assuming a user knows what digital addiction is before giving them information about how to combat or prevent it. An online questionnaire was created to assess the relationship users have with their smartphone. Survey method is used during evaluation.

During the literature survey, considerations were made into what is currently out there on the market, what the users wish to have functionality wise and what might deter users from using an application. Research went into understanding what digital addiction is and how it affects people to recognise what considerations should be made and what the requirements should be for the solution. Kanban is used in managing the time for the project as it offered good visibility on what exactly needed to be worked on and when. Agile development principles were applied during this study to ensure a successful implementation.

\section{REQUIREMENTS}

Requirements elicitation is key in requirement creation, as it shows what goals and objectives should be met by the solution. To help elicit the requirements, an analysis is done to understand what potential stakeholders would require and identify what they would want in an application that would help them see and control their phone usage.

The criteria for this research was to obtain reviews from users who have used similar applications that control their phone usage, these users were not technically stakeholders as they would not be able to interact with the system. However, this process was important to elicit the needs of users who would be interested in using an application like the proposed solution, thus the reviews were treated as stakeholder requirements to an extent. The user reviews were publicly posted by users online. Some of these reviews helped to see what sort of functionalities people liked and what they wished to be included within the application they were using. However, it is also important to mention that whilst a user may want something to be added to the solution, it is important to consider the feasibility of the feature. Also, consider if the functionality they require has any practical use that helps achieve the required goals, as well as ensuring that adding anything unnecessary into the solution is avoided. This is important as not every functionality requested by the user is something that helps achieve the overall aim of the solution.

Goal modelling was used to assist with the elicitation of the main requirements for this solution. Fig. 1 shows the goal modelling diagram which helps to visualise what tasks should be completed. In addition, it also helps elicit the nonfunctional goals that should be met alongside the functional ones. The MoSCoW method was used in order to create the list of requirements. Some selected requirements are as follows:

R1. The solution must show the user the time spent in total on their mobile phone daily.

R2. The solution must display the exact time spent of the user's most frequently used applications in a chart.

R3. The solution must display information about digital addiction to increase user awareness on the topic.

R4. The solution must allow the user to block certain applications from being used. 


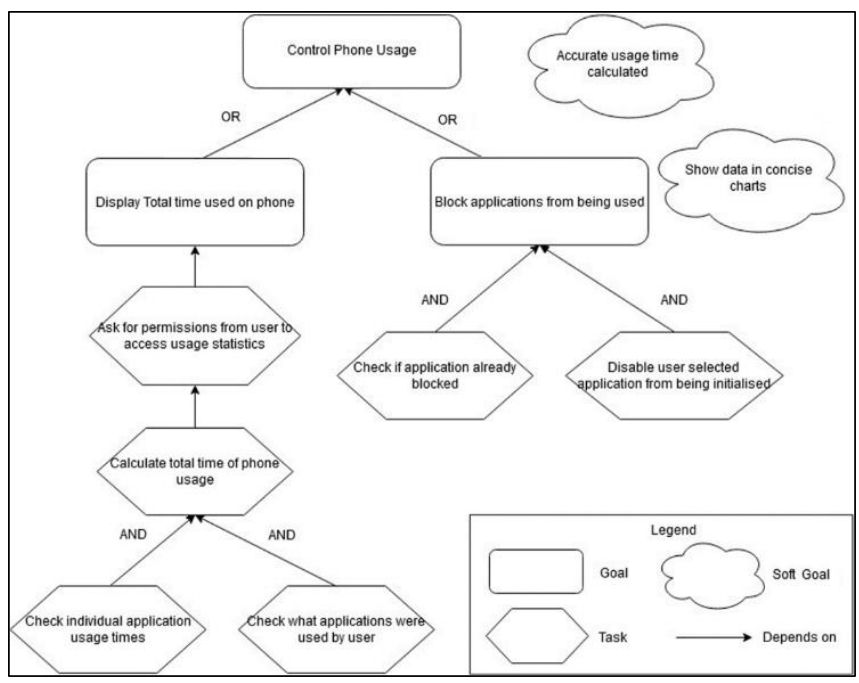

Fig. 1. Goal Modelling Diagram

R5. The solution should display a total time spent on each application per week when the user selects the application from the chart.

R6. The solution should display a counter of how many times the user has unlocked their phone that day.

R7. The solution should notify the user if they have launched a blocked application.

R8. The solution could allow the user to block an application for a predefined time that is set by the user.

Below is the list of selected non-functional requirements:

Usability: The application is designed to be used every day. It must be easy to navigate and use. Nielsen's Heuristics are considered to ensure this requirement is met.

Reliability: The application must not crash or fail. Recovering from any potential failures must be considered, and prevention methods such as validation must be included.

Supportability: Consideration must be taken to ensure as many devices can be used as possible with the application.

Performance: All features of the application must be responsive in a timely manner that does not disrupt the user.

In this research, we have considered use of gamification methods. Gamification is the means of implementing gamelike mechanics to motivate a user to use a piece of software for the means of enhancing positive patterns of use, such as increasing user activity and performance. In the literature, it has been reported that some forms of gamification can have adverse behavioural outcomes if such methods are used [14]. To alleviate any potential dangers of adverse effects when using the solution, a decision was made that gamification will not be implemented into the solution at this time due to the uncertainty of it working to a beneficial effect in the given context.

\section{DESIGN}

Designing an application for the most part is straightforward as long as requirements are fully understood and known, they will dictate what is needed to be included in the design of the solution. With Nielsen's heuristics for usability in mind, we designed some User Interface (UI) mock-ups using online software called Moqups to get an idea of the final look of the application. The system is designed in a way that the user does not spend too much time navigating around the application to complete the tasks, which is why a lot of the functionality are made available on the first page when the user launches the application. However, with that in mind, it is important to not to have too much data at one time on a page, which is why some of the important functionalities are split onto other pages. It is also important to note that smartphone screen sizes can vary so ensuring not to display too many features on one page will be better.

Charts are preferred to present the usage data. Charts can be in many different forms and designs. It would be beneficial to ensure that the design of the chart is easy to read. This is because that a user may not necessarily have too much time to look at a chart for an extended amount of time, they are on their phone and may only have a minute to look at something before they have to go back to what they are doing in real life.

While designing the application, it should be approached cautiously to ensure that the user does not feel like they are accused of having digital addiction. The intent is to allow a user to make an informed decision about their phone usage. A way to approach meeting this is through careful design and to only state accurate information from credible sources. However, as digital addiction is a new research area, there are limited sources with accurate information in the literature and there is currently no standard method of diagnosing this condition [15]. So, it is vital not to allow a user to selfdiagnose because self-diagnosis can cause heightened medical anxiety and worry in a user [16]. Hence, only non-imposing facts are stated in the 'Learn about Digital Addiction' page and any self-administering tests that can impose a selfdiagnosis are not included. The focus of the design is to ensure that all of the features are optional, so the user has free will to control their phone usage.

\section{IMPLEMENTATION}

An Android application was implemented as a proof of concept solution. Android Studio IDE was chosen for the implementation due to having previous experience with the software and Java. To implement the charts, such as the pie chart on the main page, a publicly available library called MPAndroidChart on GitHub was used. The documentation for this chart library is extensive and the charts are highly customisable.

Permission requests are important to ensure that the solution follows correct consent procedures as highlighted by the General Data Protection Regulation (GDPR) as well as inform the user that the application they are about to use does access information from other applications. To see or use information from other applications, the application needed to request Usage Stats, which is identified by the code 'OPSTR_GET_USAGE_STATS'.

For accurate calculation of usage time, UsageEvents class was used which calculates the time between the application being present in the foreground versus when it goes in the background (i.e. not being used by the user). An application called Tinycore was used alongside testing this functionality to check things like battery and CPU usages. The blocking functionality relies on controlling the behaviour of other applications, this will require running a service in the background so that the application will be able to control the blocking of other applications. 


\section{EVALUATION}

To evaluate our approach and prototype implementation we performed user testing. To best evaluate the solution, the application was put on GooglePlay to allow users to download the application on their phone and test it (Available from https://play.google.com/store/apps/details?id=com.da.digitala ddictionapp). Fig.2 shows a screenshot from the store listing whereas the application is called as Focus. There was a total of 14 testers for the application. 13 of them filled in a survey via a Google Form and 1 of them made a GooglePlay review.
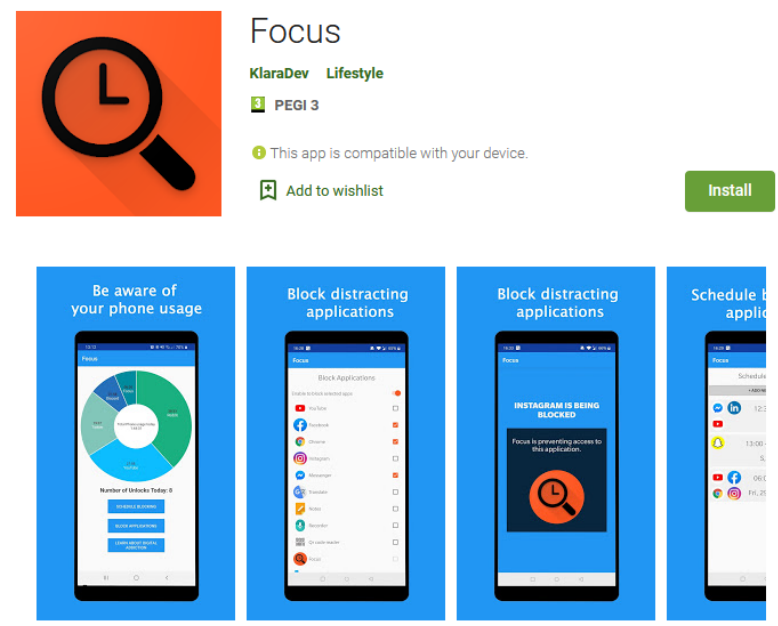

Focus lets you take control of your applications to allow you to get on with your everyday activities The aim here is to ensure that you are making informed decisions about your phone usage to help ensure you aren't using your phone in an excessive manner.

Fig. 2. GooglePlay Store Listing

Overall, the application received positive and constructive feedback through looking at the reviews based on the responses. $100 \%$ of the users have said that the application has made them feel more aware of their phone usage, which is one of the most important factors when it comes to combatting digital addiction [16]. This is because it can help users make more informed decisions about their phone usage in the future, in turn thus helping themselves use the phone less if they notice any obsessive or unhealthy patterns of use [16].

User feedback provided an essential stance as to what users would want out of this application in the future as well as how they respond to seeing their phone usage. 5 out of the 13 responses aimed towards having a parental mode or a password functionality present to assist with parents, this was the most popular request and thus is something that would be highly considered to be implemented in future work. Other responses given aimed towards wanting the blocking applications functionality to be improved.

\section{CONCLUSION}

We presented design and development of a mobile application to monitor and control phone usage. The prototype implementation allows a user to monitor and control their phone usage. The solution is ad free and does not have any features that users will have to pay for, compared to other applications that are currently on the market. The prototype implementation provides a research facility for experimental designs and data collection studies. It is still limited in the design regarding fully combatting digital addiction due to proper design guidelines and recommendations are still being discussed in the literature.
Potential future work includes implementing a parental control feature. This feature could include some authentication to change the settings like blocking certain applications. This is because some applications that are available on smartphones like certain games may prove too distracting for some children and parents might be hesitant to allow their children to use these applications. Additionally, this was the most popular choice of functionality to be requested by users from user testing. Future efforts would also include making the solution available on different platforms and operating systems to increase the potential user base for the solution. It also could mean that a user can see their usage of digital devices across their entire range of devices, as well as control different devices from just their phone alone.

\section{REFERENCES}

[1] J. Poushter, "Smartphone ownership and internet usage continues to climb in emerging economies", Pew Research Center, Report, 2016.

[2] S. Cham, J. McAlaney, and R. Ali, "Digital addiction - definition, characteristics and countermeasures", Bournemouth University Digital Addiction Research presentation. Available online from: https://www.slideshare.net/Sociad-BU/digital-addiction-and-whatyou-need-to-know. [Accessed 10 April 2020], 2018.

[3] K. Demirci, M. Akgönül, and A. Akpinar, "Relationship of smartphone use severity with sleep quality, depression, and anxiety in university students", Journal of Behavioral Addictions, 4, pp.85-92, 2015.

[4] C. Montag and P. Walla, "Carpe diem instead of losing your social mind: Beyond digital addiction and why we all suffer from digital overuse", Cogent Psychology, 3(1), 2016.

[5] M. Samaha and N. Hawi, "Relationships among smartphone addiction, stress, academic performance, and satisfaction with life", Computers in Human Behavior, 57, pp. 321-325, 2015.

[6] S. Cham, A. Algashami, M. Aldhayan, J. McAlaney, K. Phalp, M.B. Almourad, and R.Ali, "Digital addiction: negative life experiences and potential for technology-assisted solutions", In WorldCist'19 - 7th World Conference on Information Systems and Technologies, 2019.

[7] S.P. Walsh, K.M. White, and R.M. Young, "Over-connected? A qualitative exploration of the relationship between Australian youth and their mobile phones", Journal of Adolescence, 31(1), pp.77-92, 2008 .

[8] Guardian Support, "Can you ban mobile phones in the workplace?", Available online from: https://www.guardiansupport.co.uk/blog/canyou-ban-mobile-phones-in-the-workplace/ [Accessed 11 April 2020].

[9] Statista, "Percentage of households with mobile phones in the United Kingdom (UK) from 1996 to 2018", Available online from: https://www.statista.com/statistics/289167/mobile-phone-penetrationin-the-uk/ [Accessed 10 April 2020].

[10] M. Lochtefeld, M. Bohmer, and L. Ganev, "AppDetox: Helping users with mobile app addiction", In Proceedings of the 12th International Conference on Mobile and Ubiquitous Multimedia, 2013.

[11] AppInstitute, "Free vs Paid Apps: Pros \& Cons", Available from: https://appinstitute.com/free-paid-apps/, 2016.

[12] F.H. Cate and V. Mayer-Schönberger, "Notice and consent in a world of Big Data”, International Data Privacy Law, 3(2), pp.67-73, 2013.

[13] R. Ali, N. Jiang, K. Phalp, S. Muir, and J. McAlaney, "The emerging requirement for digital addiction labels", In International working conference on requirements engineering: Foundation for software quality, pp. 198-213, 2015.

[14] A.M. Toda, P.H. Valle, and S. Isotani, "The dark side of gamification: An overview of negative effects of gamification in education", Researcher links workshop: higher education for all, pp.143-156, 2017.

[15] A. Alrobai, J. McAlaney, H. Dogan, K. Phalp, and R. Ali, "Exploring the requirements and design of persuasive intervention technology to combat digital addiction", In: 6th International Working Conference on Human-Centred Software Engineering (HCSE'16), pp.130-150, 2016.

[16] A. Alrobai, J. McAlaney, K. Phalp, and R. Ali, "Exploring the risk factors of interactive e-health interventions for digital addiction", Int. Journal of Sociotechnology and Knowledge Dev., 8 (2), pp.1-15, 2016. 